\title{
Effect of a Photonic Band Gap on the Threshold and Output Power of Solid-State Lasers and Light-Emitting Diodes
}

\author{
M. H. Szymanska, A. F. Hughes, and E. R. Pike \\ Department of Physics, King's College London, Strand, London, WC2R 2LS, United Kingdom
}

(Received 20 November 1998)

\begin{abstract}
We present results for the operation of a three-level solid-state laser both with normal and with completely suppressed spontaneous emission between the lasing levels. The output power difference in these two cases drops as the pump rate is increased above threshold and is not greatly different at higher powers. The threshold pump power, although typically reduced by an order of magnitude, is not zero and our results thus throw further light on the concept of "thresholdless" lasing. The theory is also applicable to light-emitting diodes and amplifiers which function on the same principles as a laser.
\end{abstract}

PACS numbers: 42.70.Qs

There is currently considerable interest in materials with a photonic band gap (PBG), i.e., materials in which certain frequencies of light cannot propagate. The first use of the idea of a photon band structure was probably made by Ohtaka in 1979 [1]. It was, however, Yablonovitch's work of 1987 [2] that really aroused a widespread interest in this area. PBG materials have already been fabricated in three dimensions for the microwave and infrared regions, and more recently opal crystals have been used for the visible region [3]. Current research is being undertaken to fabricate PBG optical materials [4]. Two-dimensional structures at nanometer wavelengths have already been realized [5]. When an excited atom is placed in such a structure it will be prevented from emitting light if its transition frequency lies within the forbidden range. It is, however, possible to introduce a defect in such a structure so that only one particular mode can be supported. Hence PBG materials can be used to control spontaneous emission and as this is a fundamental process of the laser these materials could be used to produce new types of lasers. By introducing a defect which allows the propagation of only one mode within the forbidden region we could suppress the spontaneous emission to modes other than that particular single laser mode. In principle, we could make atoms emit only into one cavity mode which would very much reduce losses from the system and, therefore, achieve an ideal $\beta$ factor of 1 [6]. The $\beta$ factor is defined as

$$
\beta=\frac{\text { rate of spontaneous emission into lasing mode }}{\text { total rate of spontaneous emission }}
$$

and its modification has led to a discussion of thresholdless lasing [7]. Recent work concentrates on modifying spontaneous emission, and hence the $\beta$ factor, with the use of photonic wells and wires. It is expected that such a kind of laser would have a lower threshold and be more efficient than a conventional one.

The aim of this work was to obtain a theoretical lower bound on the lasing threshold of the PBG laser in order to compare its properties, such as laser threshold and output power, with the conventional laser case. To do this we assume that the lasing mode is sufficiently far from the band edges to render spontaneous emission from it to be negligible. Vats and John [8] have discussed spontaneous emission when non-Markovian band-edge influences occur.

The solid-state laser falls into the so-called class $B$ category when $\gamma_{\perp} \gg \gamma_{\|} \approx 2 \kappa$, where $\kappa$ is a cavity loss rate and $\gamma_{\perp}, \gamma_{\|}$are the polarization and inversion decay rates, respectively. There have been ever more sophisticated laser theories since the mid sixties but, unfortunately, it seems that none of them can yet satisfactorily describe the properties of class- $B$ lasers with all the spontaneous emission rates built into the model. Thus, surprisingly, none of them could be used to calculate the difference in threshold and output power for lasers with and without a photonic band gap. The well-known four-level Scully-Lamb theory of laser photon statistics $[9,10]$ does not take the spontaneous emission between the lasing levels into account at all and is essentially for class- $A$ lasers. It is the only one, however, to go to all orders in the coupling constant between the cavity modes and the lasing atoms. The quantum-mechanical Langevin equations do have spontaneous emission between the lasing levels built into the model and it is possible to obtain the threshold condition, which, in principle, then could be analyzed in the presence of a photonic band gap. However, at and close to the laser threshold no way of solving these equations is known which treats fully the fluctuations. In particular it is also not possible to calculate the photon statistics and the theory is for two-level atoms only. Lugiato's master equation method for the density operator $[11,12]$ also has spontaneous emission built into the model and all pump and decay rates are represented by explicit interactions between the lasing system and reservoirs with positive and negative absolute temperatures, respectively, but again the theory is for two-level atoms only and only valid below threshold. These equations were solved by Lugiato using the Glauber diagonal $P$ representation [12]. Other authors have solved this model by transforming into a Fokker-Planck equation 
[13-15]. However, all these results have a very complicated form which allows fluctuations to be calculated only numerically, and it is still not possible to obtain the photon statistics $p_{n}$.

We have improved these theories sufficiently for our purpose by starting from Lugiato's two-level-atom model but by avoiding his use of a "smoothing" approximation (see also the derivation of the Scully-Lamb theory from the Lugiato model described in Pike and Sarkar [15]) and then explicitly solving the resulting equations in the photonnumber representation. We have also been able to solve the three-level model and to take the theory to all orders in the coupling constant between the cavity modes and the lasing atoms in both the two- and three-level cases.

We will present the solution for the three-level lasing model to all orders in the coupling constant. The threelevel model is a more realistic scenario and we want to compare our work to that which has discussed the concept of thresholdless lasing [7]. It also appears that the twolevel model has unrealistic behavior far above threshold and this problem is removed using three levels. The atomic-level structure and the various transitions used in our calculations are shown in Fig. 1.

In the three-level laser model all elements connected with the laser operation are divided into two parts, a small system $S$ and a large heat bath $B$. S $S$ will be described by a Hamiltonian $H_{S}$, the bath by $H_{B}$, and the interaction between $B$ and $S$ by $H_{S B}$. The system contains $N$ threelevel transitions, a single electromagnetic field mode and the lasing transition-field interaction. Both the three-level transitions and field are treated quantum mechanically, and the interaction is taken in the rotating wave approximation. The bath can be divided into three parts: a field bath which represent losses of the laser mode from the cavity through the partially transmitting output mirror, a bath which represents the interactions of the excited levels with the vacuum modes, i.e., spontaneous emission to modes other than the lasing mode, and a bath which represents pumping from the lowest level to the highest one.

For a semiconductor laser we have an analogy with the three-level model. The gap in a semiconductor material between the conduction and valence bands is where the lasing action occurs. A flow of holes in the valence band corresponds to the depletion of the lower lasing levels [16]. Precise Hamiltonians for the baths are not known but this is not very important since we are only interested in the system behavior. It is then enough to know the Hamiltonian of the bath-system interaction. We model the baths as sets of harmonic oscillators at temperature $T$. The spontaneous emission bath is modeled as a set of oscillators at temperature $T=0 \mathrm{~K}$ and the pumping bath as a set of oscillators at negative temperature $T=-0 \mathrm{~K}$. The field bath is modeled as a set of harmonic oscillators at temperature $T$. When we are not interested in thermal photons coming back into the laser we may set this temperature to $0 \mathrm{~K}$ (this is like saying that the environment of the laser has zero temperature). The rates of exchange of energy between these baths and the system are expressed by the separate coupling constants of the interactions. The three types of baths have different influences on the system, but equilibrium between them will be reached and the system will then have a temperature (i.e., inversion) depending on the coupling constants of the two system baths and the lasing transitionlasing mode interaction. The system Hamiltonian is given by

$$
H_{S}=H_{T}+H_{F}+H_{T F}
$$

where

$$
\begin{aligned}
H_{T} & =\frac{1}{2} \sum_{j} \hbar \omega_{o} S_{j}^{3} \\
H_{F} & =\hbar \omega_{k} a_{\mathbf{k}}^{\dagger} a_{\mathbf{k}} \\
H_{T F} & =i \sum_{j=1}^{N} \hbar g\left(e^{-i \mathbf{k} \cdot \mathbf{x}_{j}} a_{\mathbf{k}}^{\dagger} S_{j 23}^{-}-e^{i \mathbf{k} \cdot \mathbf{x}_{j}} a_{\mathbf{k}} S_{j 23}^{+}\right) .
\end{aligned}
$$

$S_{j 23}^{+}, S_{j 23}^{-}$are creation and annihilation operators, respectively, between lasing levels (levels 2 and 3 in Fig. 1). The bath-system interaction Hamiltonian, $H_{S B}$, includes the decay of excited levels to off-resonant modes $\Gamma_{j 12 \downarrow}$, $\Gamma_{j 13 \downarrow}, \Gamma_{j 23}$, the pumping of the atoms to the excited state, $\Gamma_{j \dagger}$, and the decay of the resonant mode. The Hamiltonian is

$$
\begin{aligned}
H_{S B}= & \hbar\left(a_{\mathbf{k}} \Gamma_{F}^{\dagger}+a_{\mathbf{k}}^{\dagger} \Gamma_{F}\right)+\sum_{j=1}^{N} \hbar\left(S_{j 12}^{-} \Gamma_{j 12 \downarrow}^{\dagger}+S_{j 13}^{-} \Gamma_{j 13 \downarrow}^{\dagger}+S_{j 23}^{-} \Gamma_{j 23 \downarrow}^{\dagger}+S_{j 13}^{+} \Gamma_{j \dagger}\right) \\
& +\sum_{j=1}^{N} \hbar\left(S_{j 13}^{-} \Gamma_{j \uparrow}^{\dagger}+S_{j 12}^{+} \Gamma_{j 12 \downarrow}+S_{j 13}^{+} \Gamma_{j 13 \downarrow}+S_{j 23}^{+} \Gamma_{j 23 \downarrow}\right) .
\end{aligned}
$$

Thus the quantum-optical master equation takes the form

$$
\begin{aligned}
\frac{\partial \rho_{S}}{\partial t}= & -i\left[H_{S}, \rho_{S}\right]+\sum_{j=1}^{N} \frac{\gamma_{23 \downarrow}}{2}\left(\left[S_{j 23}^{-}, \rho_{S} S_{j 23}^{+}\right]+\left[S_{j 23}^{-} \rho_{S}, S_{j 23}^{+}\right]\right)+\sum_{j=1}^{N} \frac{\gamma_{13 \downarrow}}{2}\left(\left[S_{j 13}^{-}, \rho_{S} S_{j 13}^{+}\right]+\left[S_{j 13}^{-} \rho_{S}, S_{j 13}^{+}\right]\right) \\
& +\sum_{j=1}^{N} \frac{\gamma_{12 \downarrow}}{2}\left(\left[S_{j 12}^{-}, \rho_{S} S_{j 12}^{+}\right]+\left[S_{j 12}^{-} \rho_{S}, S_{j 12}^{+}\right]\right)+\sum_{j=1}^{N} \frac{\gamma_{\uparrow}}{2}\left(\left[S_{j 13}^{+}, \rho_{S} S_{j 13}^{-}\right]+\left[S_{j 13}^{+} \rho_{S}, S_{j 13}^{-}\right]\right) \\
& +\kappa\left(2 a_{\mathbf{k}} \rho_{S} a_{\mathbf{k}}^{\dagger}-a_{\mathbf{k}}^{\dagger} a_{\mathbf{k}} \rho_{S}-\rho_{S} a_{\mathbf{k}}^{\dagger} a_{\mathbf{k}}\right)=\Lambda \rho_{S}(t) .
\end{aligned}
$$




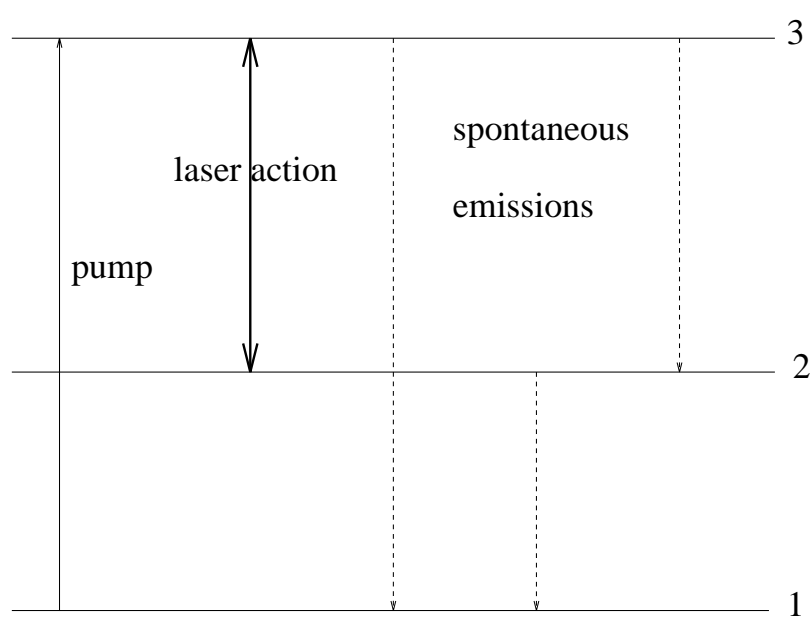

FIG. 1. Atomic-level structure and various transitions in the three-level-atom model.

Taking the trace over the transition levels, using the dilute-atom approximation and employing the Markov approximation gives us an equation for $p_{n}$, the probability of finding $n$ photons in the cavity mode, when we work in the photon-number representation. Multiplying the above expression by $S_{23}^{-} S_{23}^{+}$and $S_{13}^{-} S_{13}^{+}$, respectively, we obtain two more coupled equations. When we consider these three equations in the steady state and perform detailed balancing we find

$$
\begin{gathered}
2 \kappa p_{n}(t)=-\frac{A}{1+n B / A}\left[p_{n 2}(t)-p_{n-1,3}(t)\right], \\
\frac{\frac{2 g^{2} n}{\gamma}}{1+n B / A}\left(p_{n-1,3}-p_{n, 2}\right)-\gamma_{12 \downarrow} p_{n, 2}+\gamma_{23 \downarrow} p_{n, 3}= \\
\gamma_{13 \downarrow} p_{n, 3}+\gamma_{12 \downarrow} p_{n, 2}-\gamma_{\uparrow} p_{n, 1}=0,
\end{gathered}
$$

where

$$
\gamma=\frac{1}{2}\left(\gamma_{23 \downarrow}+\gamma_{13 \downarrow}+\gamma_{12 \downarrow}\right) .
$$

We also know that

$$
p_{n}=p_{n 1}+p_{n 2}+p_{n 3},
$$

where

$$
\begin{aligned}
p_{n}(t) & =\left\langle n\left|\rho_{F}^{I}(t)\right| n\right\rangle, \\
p_{n, 1}(t) & =\left\langle n\left|S_{13}^{-} S_{13}^{+}(t) \rho_{S}^{I}(t)\right| n\right\rangle=\left\langle 1, n\left|\rho_{S}^{I}(t)\right| n, 1\right\rangle, \\
p_{n, 2}(t) & =\left\langle n\left|S_{23}^{-} S_{23}^{+}(t) \rho_{S}^{I}(t)\right| n\right\rangle=\left\langle 2, n\left|\rho_{S}^{I}(t)\right| n, 2\right\rangle, \\
p_{n, 3}(t) & =\left\langle n\left|S_{23}^{+} S_{23}^{-}(t) \rho_{S}^{I}(t)\right| n\right\rangle=\left\langle 3, n\left|\rho_{S}^{I}(t)\right| n, 3\right\rangle .
\end{aligned}
$$

Solving the above equations we obtain the following recursion relation:

$$
p_{n}(t)=\frac{A\left[N \gamma_{\uparrow} \gamma_{12 \downarrow}-2 \kappa(n-1)\left(\gamma_{12 \downarrow}+\gamma_{\uparrow}\right)\right] p_{n-1}}{\left[2 \kappa N(1+n B / A)\left(\gamma_{13 \downarrow} \gamma_{12 \downarrow}+\gamma_{\uparrow} \gamma_{12 \downarrow}+\gamma_{12 \downarrow} \gamma_{23 \downarrow}+\gamma_{23 \downarrow} \gamma_{\uparrow}\right)+A\left(N \gamma_{23 \downarrow} \gamma_{\uparrow}+2 \kappa n \gamma_{13 \downarrow}+2 \kappa n \gamma_{\uparrow}\right)\right]},
$$

where

$$
\begin{aligned}
A & =\frac{2 N g^{2}}{\gamma}, \\
B / A & =4 g^{2} / \gamma^{2} .
\end{aligned}
$$

Thus it is possible to express $p_{n}$ as a product of $n$ elements and to calculate the average and the most probable number of photons in the cavity. The photon statistics determines the coherence of the output. In the region above laser threshold the most probable number of photons is greater than zero and in the region below and at the laser threshold is equal to zero. Thus a physical insight into our results for $\beta=1$ is given by the realization that, although always increasing linearly with input power, the cavity output is incoherent until the former condition on the photon statistics is achieved. In order to find the laser threshold we therefore need to calculate the most probable number of photons in the lasing mode. This becomes equal to the average far above threshold, where the photon statistics are symmetrical and approach the Poisson distribution. It can be shown that the most probable number of photons in the cavity is equal to

$$
\begin{aligned}
n_{p}= & \frac{\frac{2 g^{2}}{\gamma} N \gamma_{\uparrow}\left(\gamma_{12 \downarrow}-\gamma_{23 \downarrow}\right)-\frac{2 g^{2}}{\gamma} 2 \kappa\left(\gamma_{13 \downarrow}+\gamma_{\uparrow}\right)}{\frac{2 g^{2} 2 \kappa}{\gamma}\left(\gamma_{\downarrow 12}+2 \gamma_{\uparrow}+\gamma_{\downarrow 13}\right)+\frac{2 \kappa B}{A}\left(\gamma_{\downarrow 13} \gamma_{\downarrow 12}+\gamma_{\uparrow} \gamma_{\downarrow 12}+\gamma_{\downarrow 12} \gamma_{\downarrow 23}+\gamma_{\downarrow 23} \gamma_{\uparrow}\right)} \\
& -\frac{2 \kappa(1+B / A)\left(\gamma_{13 \downarrow} \gamma_{12 \downarrow}+\gamma_{\uparrow} \gamma_{12 \downarrow}+\gamma_{12 \downarrow} \gamma_{23 \downarrow}+\gamma_{23 \downarrow} \gamma_{\uparrow}\right)}{\frac{2 g^{2} 2 \kappa}{\gamma}\left(\gamma_{12 \downarrow}+2 \gamma_{\uparrow}+\gamma_{13 \downarrow}\right)+\frac{2 \kappa B}{A}\left(\gamma_{13 \downarrow} \gamma_{12 \downarrow}+\gamma_{\uparrow} \gamma_{12 \downarrow}+\gamma_{12 \downarrow} \gamma_{23 \downarrow}+\gamma_{23 \downarrow} \gamma_{\uparrow}\right)} .
\end{aligned}
$$

We can now calculate the threshold pump rate from the condition that the most probable number of photons in the lasing mode must be greater than zero.

$$
\gamma_{\uparrow}=\frac{2 g^{2} \kappa \gamma_{13 \downarrow}+\kappa \gamma_{12 \downarrow} \gamma\left(\gamma_{13 \downarrow}+\gamma_{23 \downarrow}\right)}{N g^{2}\left(\gamma_{12 \downarrow}-\gamma_{23 \downarrow}\right)-2 \kappa g^{2}-\gamma \kappa\left(\gamma_{12 \downarrow}+\gamma_{23 \downarrow}\right)} .
$$

When spontaneous emission between the lasing levels can be suppressed then $\gamma_{23 \downarrow}=0$. Recall that in the devel- opment of the theory we employed a Markov approximation. This approximation is still valid even though we use a PBG material as we are positioning our transition levels and defect mode at the center of the gap, therefore excluding any gap-edge influences. We compare characteristics for lasers with and without a photonic band gap $\left(\gamma_{23 \downarrow}=0\right)$ for a laser with parameters [17] $N=10^{7} / 2, g=10^{5}, 2 \kappa=10^{7}, \gamma_{13 \downarrow}=10^{6}, \gamma_{23 \downarrow}=$ $10^{7} / 2$, and $\gamma_{12 \downarrow}=10^{10}$, in Fig. 2. 

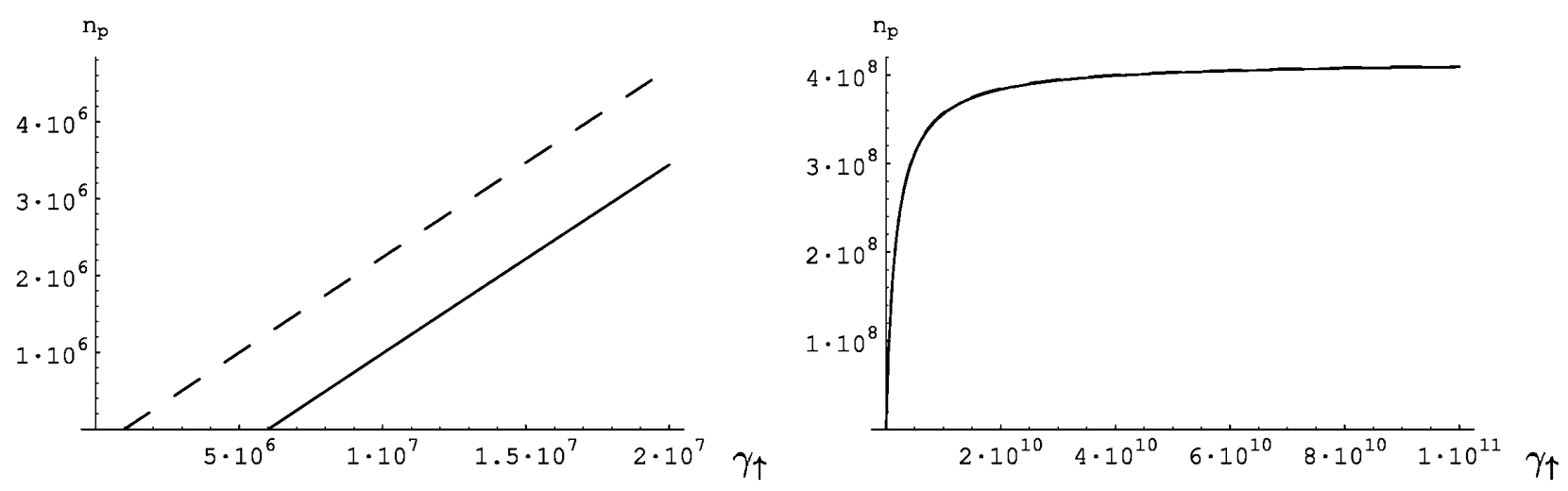

FIG. 2. The most probable number of photons as a function of pumping rate for a three-level-lasing model for lasers with (higher curve) and without PBG. The curves are indistinguishable in the second diagram.

For the case of such a PBG laser the threshold takes place at a pump rate one order of magnitude smaller than that for the case without a PBG. However, the maximum output far above threshold of both types of laser is similar. The PBG laser has a threshold pump rate given by the expression

$$
\gamma_{\uparrow, \mathrm{PBG}}=\frac{2 g^{2} \kappa \gamma_{13 \downarrow}+\kappa \gamma_{12 \downarrow} \gamma \gamma_{13 \downarrow}}{N g^{2} \gamma_{12 \downarrow}-2 \kappa g^{2}-\gamma \kappa \gamma_{12 \downarrow}} .
$$

The important point to note for the value of this pump rate is that the threshold is not zero when $\beta=1$. As for a conventional laser, the output is at first incoherent. This differs from the results of Rice et al. [7] where they find a thresholdless laser in this case. We can, in fact, directly compare our work to that of Rice et al. Their model is two-level but the lower level rapidly depletes to a lower lying unspecified state. In our model this corresponds to $\gamma_{13 \downarrow}$ being very small and $\gamma_{12 \downarrow}$ very large. With these conditions we see from Eq. (8) that $p_{n, 2}$ is then very small, which implies such a rapidly depleting lower level. The threshold of the PBG laser will then approach zero.

Our new laser theory thus allows the differences between an ideal PBG laser and a conventional laser to be calculated when the numerical values of the relevant parameters are known. A real PBG laser will not have all the spontaneous emission suppressed but our results give an upper bound on the possible improvements in performance. As would be expected, the threshold pump rate is lower. In the region of saturation the stimulated emission dominates the spontaneous emission therefore rendering it negligible. Therefore, when the $\beta$ factor is 1 , there is little increase in the output power far above threshold and the advantages are marginal at these higher powers. However, when considering LED's we are concerned with lower output powers and in this region we conclude that the output power will indeed be greatly increased due to the use of a PBG material. We are considering an extension of the theory to describe laser amplifiers, where PBG materials are also expected to improve performance significantly.

[1] K. Ohtaka, Phys. Rev. B 19, 5057 (1979).

[2] E. Yablonovitch, Phys. Rev. Lett. 58, 2059 (1987).

[3] S. G. Romanov et al., Appl. Phys. Lett. 70, 2091 (1997).

[4] J. Rarity and C. Weisbuch, in Microcavities and Photonic Bandgaps: Physics and Applications, NATO ASI, Ser. E, Vol. 324 (Kluwer, Dordrecht, 1996).

[5] T.F. Krauss et al., Nature (London) 383, 699 (1996).

[6] T. Baba et al., IEEE J. Quantum. Electron. 27, 1347 (1991).

[7] P. R. Rice and H.J. Carmichael, Phys. Rev. A 50, 4318 (1994).

[8] N. Vats and S. John, Phys. Rev. A 58, 4168 (1998).

[9] M. Scully and W.E. Lamb, Jr., Phys. Rev. 159, 208 (1967).

[10] M. O. Scully and M.S. Zubairy, Quantum Optics (Cambridge University Press, Cambridge, England, 1997).

[11] L. A. Lugiato, Physica (Amsterdam) 81A, 565 (1975).

[12] L. A. Lugiato, Physica (Amsterdam) 82A, 1 (1976).

[13] C. W. Gardiner, Quantum Noise (Springer-Verlag, Berlin, 1991).

[14] H. Haken, Light (North-Holland Publishing, Amsterdam, 1985), Vol. 2.

[15] E. R. Pike and Sarben Sarkar, The Quantum Theory of Radiation (Oxford Science Publications, Oxford, England, 1995).

[16] C. Kittel, Introduction to Solid State Physics (John Wiley and Sons, New York, 1986).

[17] R. Loudon et al., Phys. Rev. A 48, 681 (1993). 